\title{
Hemolysis-associated endothelial dysfunction mediated by accelerated NO inactivation by decompartmentalized oxyhemoglobin
}

\author{
Peter C. Minneci, ${ }^{1,2,3}$ Katherine J. Deans, ${ }^{1,3}$ Huang Zhi, ${ }^{4}$ Peter S.T. Yuen, ${ }^{5}$ \\ Robert A. Star, ${ }^{5}$ Steven M. Banks, ${ }^{1}$ Alan N. Schechter, ${ }^{2}$ Charles Natanson, ${ }^{1}$ \\ Mark T. Gladwin, ${ }^{1,4}$ and Steven B. Solomon ${ }^{1}$
}

\begin{abstract}
${ }^{1}$ Critical Care Medicine Department, NIH, Bethesda, Maryland, USA. ${ }^{2}$ Molecular Medicine Branch, NIDDK, NIH, Bethesda, Maryland, USA. ${ }^{3}$ Department of Surgery, Massachusetts General Hospital, Boston, Massachusetts, USA. ${ }^{4}$ Vascular Medicine Branch, National Heart, Lung, and Blood Institute (NHLBI), NIH, Bethesda, Maryland, USA. ${ }^{5}$ Renal Diagnostics and Therapeutics Unit, NIDDK, NIH, Bethesda, Maryland, USA.
\end{abstract}

\begin{abstract}
During intravascular hemolysis in human disease, vasomotor tone and organ perfusion may be impaired by the increased reactivity of cell-free plasma hemoglobin $(\mathrm{Hb})$ with NO. We experimentally produced acute intravascular hemolysis in a canine model in order to test the hypothesis that low levels of decompartmentalized or cell-free plasma $\mathrm{Hb}$ will severely reduce NO bioavailability and produce vasomotor instability. Importantly, in this model the total intravascular $\mathrm{Hb}$ level is unchanged; only the compartmentalization of $\mathrm{Hb}$ within the erythrocyte membrane is disrupted. Using a full-factorial design, we demonstrate that free waterinduced intravascular hemolysis produces dose-dependent systemic vasoconstriction and impairs renal function. We find that these physiologic changes are secondary to the stoichiometric oxidation of endogenous NO by cell-free plasma oxyhemoglobin. In this model, $80 \mathrm{ppm}$ of inhaled NO gas oxidized $85-90 \%$ of plasma oxyhemoglobin to methemoglobin, thereby inhibiting endogenous NO scavenging by cell-free $\mathrm{Hb}$. As a result, the vasoconstriction caused by acute hemolysis was attenuated and the responsiveness to systemically infused NO donors was restored. These observations confirm that the acute toxicity of intravascular hemolysis occurs secondarily to the accelerated dioxygenation reaction of plasma oxyhemoglobin with endothelium-derived NO to form bioinactive nitrate. These biochemical and physiological studies demonstrate a major role for the intact erythrocyte in NO homeostasis and provide mechanistic support for the existence of a human syndrome of hemolysis-associated NO dysregulation, which may contribute to the vasculopathy of hereditary, acquired, and iatrogenic hemolytic states.
\end{abstract}

\section{Introduction}

$\mathrm{NO}$ is continuously synthesized from endothelium and regulates homeostatic vascular functions such as vasodilation, inhibition of platelet activation and thrombosis, inhibition of endothelial adhesion molecule and endothelin-1 expression, and modulation of intimal and smooth muscle proliferation (1-5). A paradox and controversy in vascular biology surrounds the near diffusion-limited dioxygenation reaction of $\mathrm{NO}$ with oxyhemoglobin that oxidizes NO to nitrate, an inactive metabolite (Reaction 1).

\section{Reaction 1}

$\mathrm{NO}+\mathrm{HbFe}^{\mathrm{Il}}-\mathrm{O}_{2} \rightarrow \mathrm{NO}_{3}^{-}+\mathrm{HbFe}^{\prime \prime \prime}$,

where $\mathrm{HbFe}^{\mathrm{II}}-\mathrm{O}_{2}$ is ferrous oxyhemoglobin and $\mathrm{HbFe}^{\mathrm{III}}$ is methemoglobin.

The rapid $\left(10^{7} \mathrm{M}^{-1} \mathrm{~s}-1\right)$ and irreversible nature of this reaction in the context of an extremely high concentration of intravascular oxyheme groups $(10,000 \mu \mathrm{M})$ suggests that endothe-

Nonstandard abbreviations used: CI, cardiac index; CVP, central venous pressure; D5W, 5\% dextrose water; $\mathrm{Hb}$, hemoglobin; $\mathrm{HbFe}^{\mathrm{II}} \mathrm{O}_{2}$, ferrous oxyhemoglobin; MAP, mean arterial pressure; PAP, pulmonary artery pressure; PCWP, pulmonary capillary wedge pressure; SVRI, systemic vascular resistance index.

Conflict of interest: The authors have declared that no conflict of interest exists.

Citation for this article: J. Clin. Invest. 115:3409-3417 (2005).

doi:10.1172/JCI25040. lium-derived NO should be incapable of autocrine diffusion to smooth muscle secondary to rapid inactivation (6-9). Indeed, even hemoglobin ( $\mathrm{Hb}$; at $6 \mu \mathrm{M}$ oxyheme) in buffer completely inhibits endothelium-dependent blood flow in aortic ring bioassays and produces vasoconstriction in vivo (10). It is believed that the compartmentalization of $\mathrm{Hb}$ within the erythrocyte limits this inactivation reaction. In vitro studies suggest that combined NO diffusional barriers along the endothelium in laminar flowing blood (11-13), in the unstirred layer around the erythrocyte $(14,15)$, and in the submembrane protein scaffolding of the erythrocyte $(16,17)$ reduce the reaction rate of $\mathrm{NO}$ with intracellular $\mathrm{Hb}$ by 100 - to 1,000 -fold. In addition to the formation of diffusional barriers, the erythrocyte limits $\mathrm{Hb}$ extravasation into the extracellular space (10).

While a physiological role for the erythrocyte in modulating the NO-Hb reaction is supported by the observed vasoconstrictive effects and systemic toxicity of cell-free, $\mathrm{Hb}$-based blood substitutes $(6,7)$, and is consistent with the endothelial dysfunction recently described in sickle cell disease $(18,19)$, the idea that hemolysis producing levels of plasma $\mathrm{Hb}$ of only $20-150 \mu \mathrm{M}$ (heme concentration) can significantly affect vascular physiology is not universally accepted. Indeed, a role for $\mathrm{Hb}$ in mediating NO scavenging and for the erythrocyte in limiting this process has been challenged on conceptual grounds (20-22). In addition, the primary mechanism for the vasoactivity of cell-free $\mathrm{Hb}$-based blood substitutes remains controversial and has been 
A
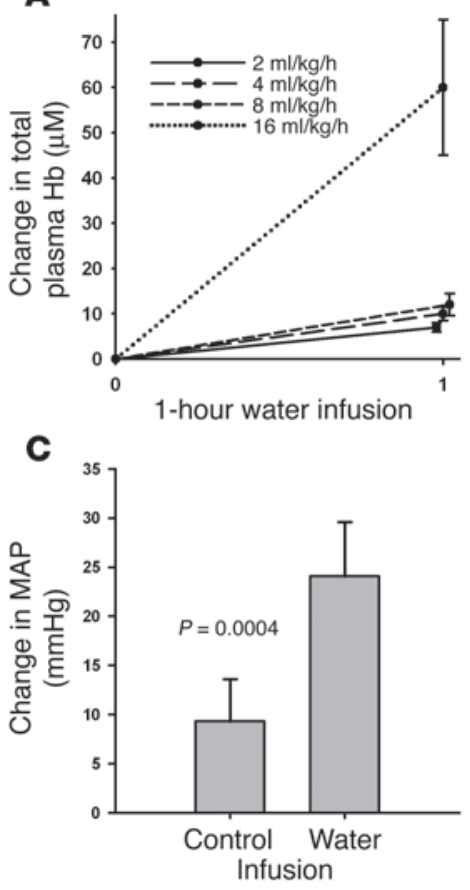

B

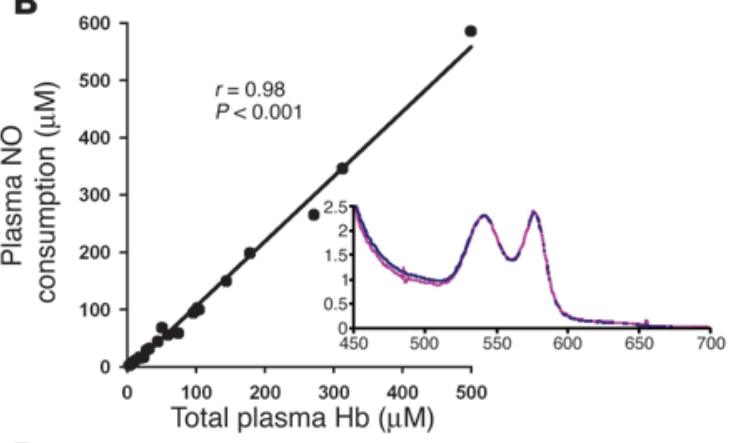

D

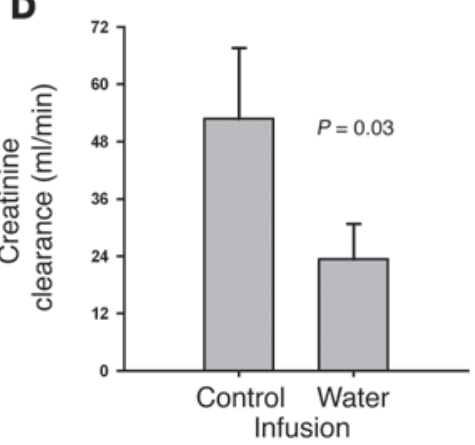

Figure 1

Characteristics of the canine free water hemolysis model. (A) Increasing rates of free water infusion caused increased rates of hemolysis with higher total plasma $\mathrm{Hb}$ levels (concentration in terms of heme groups). (B) The amount of plasma $\mathrm{Hb}$ (concentration in terms of heme groups) released by free water-induced intravascular hemolysis remained ferrous $\left(\mathrm{HbFell}_{-} \mathrm{O}_{2}\right)$ and correlated with the ability of the plasma to consume NO in a $1: 1$ stoichiometric ratio $(r=0.98 ; P<0.001)$. The inset represents a sample spectrum of the $\mathrm{Hb}$ species in the plasma, which demonstrates that the cell-free plasma $\mathrm{Hb}$ consists predominantly of oxyhemoglobin. (C and $\mathbf{D})$ Compared with 6-hour control infusions (D5W and normal saline combined), 6-hour infusions of free water caused a significant increase in MAP $(\mathbf{C} ; P=0.0004)$ and a significant decrease in 6-hour creatinine clearance $(\mathbf{D} ; P=0.03)$ from time 0 . attributed to both NO scavenging (23) and the premature delivery of oxygen to the systemic arterioles $(24,25)$, both of which mediate vasoconstriction.

In response to the uncertainty surrounding the relationship between $\mathrm{Hb}$ decompartmentalization and $\mathrm{NO}$ consumption, we have developed a canine model of intravascular hemolysis to test 3 major hypotheses: (a) simple cellular compartmentalization of $\mathrm{Hb}$ within the erythrocyte greatly limits the dioxygenation reaction of $\mathrm{Hb}$ with endothelially derived $\mathrm{NO}$; (b) intravascular hemolysis produces a state of resistance to endogenously produced and exogenously delivered NO; and (c) inhaled NO gas will selectively oxidize the plasma $\mathrm{HbFe}^{\mathrm{II}}-\mathrm{O}_{2}$ to methemoglobin, which will inhibit endogenous $\mathrm{NO}$ consumption and systemic vasoconstriction during hemolysis.

\section{Results}

Free water-induced intravascular hemolysis produces dose-and time-dependent increases in plasma $\mathrm{HbFe}^{I I-\mathrm{O}_{2}}$, which stoichiometrically consumes $\mathrm{NO}$ and impairs systemic and renal perfusion. Free water-induced hemolysis produces direct intravascular hemolysis, thereby maintaining the same intravascular concentration of $\mathrm{Hb}$ during hemolysis. Only the cellular compartmentalization of the $\mathrm{Hb}$ is disrupted in these experiments. Model-building experiments demonstrated that the amount of intravascular hemolysis was dependent on the rate and duration of free water infusion (Figure 1A). Infusion rates of $16 \mathrm{ml} / \mathrm{kg} / \mathrm{h}$ produced steady rises in plasma $\mathrm{Hb}$ to concentrations of 200-300 $\mu \mathrm{M}$ (concentration in terms of heme) over a 6-hour infusion. During hemolysis, total $\mathrm{Hb}$ levels were unchanged and hematocrit (Hct) was unchanged, reflecting the fact that levels of plasma $\mathrm{Hb}$ of $200 \mu \mathrm{M}$ represent hemolysis of only $2 \%$ of all erythrocytes. In this model, cell-free plasma $\mathrm{Hb}$ released from the erythrocytes remained $92 \% \mathrm{HbFe}^{\mathrm{II}}-\mathrm{O}_{2}$, and the plasma oxyhemoglobin concentration (in terms of heme) correlated with increased plasma NO consumption in a 1:1 stoichiometric ratio (Figure 1B).
In paired 6-hour pilot experiments, the physiologic responses to $5 \%$ dextrose water (D5W) and normal saline were similar ( $P=\mathrm{NS}$ ) and produced no measurable intravascular hemolysis. D5W was chosen as the control fluid for the full-factorial design to control for the hypotonic effects of free water. Consistent with the observed stoichiometric consumption of $\mathrm{NO}$ by plasma $\mathrm{Hb}$ produced during free water infusion (Figure 1B), free water infusion produced significant increases in mean arterial pressure (MAP)

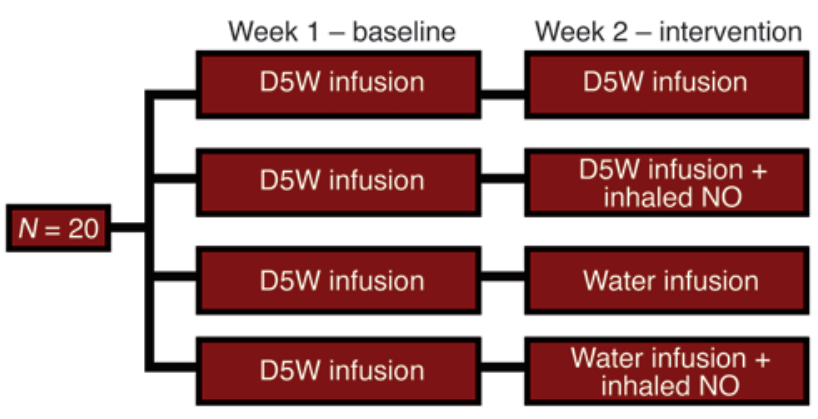

Figure 2

Full-factorial study design of the effects of intravascular hemolysis and inhaled NO. In order to minimize variability and limit the number of animals necessary to perform these studies, each animal underwent a baseline and intervention experiment. During the first week, each animal underwent a 6-hour baseline study with an infusion of D5W to control for the effects of the fluid challenge. During the second week, animals underwent a 6-hour intervention study in which they were randomized to receive 1 of 4 treatments (D5W; D5W plus inhaled NO; free water; or free water plus inhaled NO). This design allowed for the comparison of differences across treatment groups by subtracting calculated differences within animals (from baseline to intervention) in each treatment group. Comparison of these differences of the differences allowed for analysis of the effects of hemolysis, the effects of inhaled $\mathrm{NO}$, and detection of any interaction between the 2 interventions. 

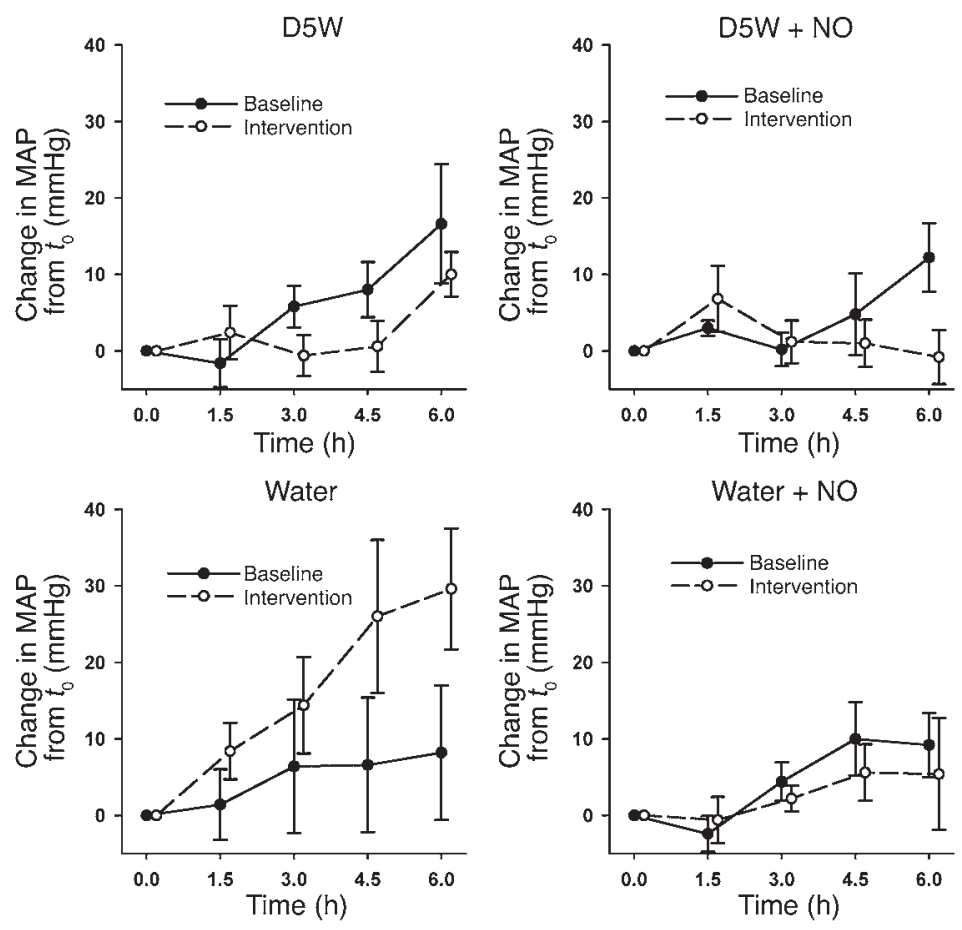

Figure 3

Effects of hemolysis and inhaled NO on MAP. In paired experiments, all animals received a 6-hour D5W infusion during the baseline study and 1 week later were randomized to a 6-hour intervention study of either D5W, D5W plus NO, free water, or free water plus NO. Changes in MAP over the course of the 6-hour baseline (filled circles) and intervention studies (open circles) are shown. In all 4 groups of animals, there were statistically similar small increases in MAP during the 6-hour baseline D5W infusion. Compared with an equivalent infusion of D5W with or without NO (nonhemolyzing control groups), free water-induced intravascular hemolysis caused a significant increase in MAP, which was attenuated by the concurrent inhalation of $\mathrm{NO}$ gas ( $P=0.0003$ for interaction of $\mathrm{NO}$ and hemolysis).

(Figure 1C) and a significant decrease in creatinine clearance over a 6-hour infusion (Figure 1D) compared with control infusions (D5W and normal saline combined).

Effects of hemolysis and inhaled NO on cardiovascular physiology and end-organ function. In order to test the effects of intravascular hemolysis on systemic perfusion and the role of $\mathrm{Hb}$-based NO scavenging as a mechanism for hemodynamic perturbations, full-factorial experiments were performed using 4 experimental interventions (Figure 2). In these experiments, hemolysis produced increases in MAP and systemic vascular resistance index (SVRI) and a decrease in cardiac index (CI). Consistent with this effect being mediated by NO scavenging by plasma oxyhemoglobin, there was a significant interaction between the presence or absence of hemolysis and the effects of inhaled NO on MAP $(P=0.0003$ for interaction of $\mathrm{NO}$ and hemolysis; Figures 3 and 4), SVRI ( $P=0.0003$ for interaction of NO and hemolysis; Figure 4), and CI $(P=0.02$ for interaction of NO and hemolysis; data not shown). Inhaled NO attenuated hemolysis-induced increases in MAP and SVRI, and hemolysis induced decreases in CI. In contrast, inhaled NO did not change hemodynamic parameters in nonhemolyzing animals. These data indicate that the effects of inhaled NO during hemolysis were secondary to the direct attenuation of the vasomotor effects of cell-free plasma $\mathrm{Hb}$ on the vasculature and not to inhaled NOinduced systemic vasodilatation.

The observed increases in total plasma Hb levels were similar in both groups of hemolyzing animals with and without NO gas inhalation. However, the relationship between increasing MAP and total plasma $\mathrm{Hb}$ concentration, and SVRI and total plasma $\mathrm{Hb}$, differed based on the administration of NO gas $(P=0.003$ and $P=0.001$ respectively; Figure 4). Thus, as total plasma $\mathrm{Hb}$ levels increased, the associated increase in MAP and SVRI was blunted with NO gas inhalation. These results reveal that exogenous NO attenuates the hemodynamic vasopressor effects of hemolysis without changing cell-free $\mathrm{Hb}$ levels. This is consistent with an inhibition of the ability of circulating $\mathrm{Hb}$ to scavenge systemic $\mathrm{NO}$ in the presence of inhaled NO.

Hemolysis also reduced the ability of the kidneys to compensate for infusion-related hyponatremia and led to a decrease in creatinine clearance. This renal dysfunction was attenuated by inhaled NO $(P=0.04$ for interaction of $\mathrm{NO}$ and hemolysis and $P=0.01$ for ordering of the 4 treatment groups; Figure 5, A and B).

In animals receiving free water infusion (with and without NO gas inhalation), plasma haptoglobin levels rapidly decreased to less than $33 \%$ of baseline values during the 6-hour infusion (measured by Western blotting; data not shown).

Vasopressor effects of hemolysis are mediated by the dioxygenation reaction of plasma oxybemoglobin with $N O$ and inhibited by conversion of plasma oxyhemoglobin to methemoglobin during NO inhalation. During free water-induced hemolysis, plasma NO consumption increased in an approximate 1:1 ratio with total plasma Hb levels (Figure 6A). Spectral deconvolution of the plasma $\mathrm{Hb}$ species in hemolyzing animals demonstrated that the plasma $\mathrm{Hb}$ was $92 \%$ $\mathrm{HbFe}^{\mathrm{II}}-\mathrm{O}_{2}$. In contrast, in hemolyzing animals treated with inhaled NO, there was limited plasma NO consumption despite increasing total $\mathrm{Hb}$ levels. Spectral deconvolution of plasma in these animals revealed that the plasma $\mathrm{Hb}$ consisted of predominantly methemoglobin in hemolyzing animals receiving inhaled NO. Therefore, inhaled NO attenuated the physiologic effects of free water-induced intravascular hemolysis by altering the ability of the plasma $\mathrm{Hb}$ to react with endogenous NO. During hemolysis, oxyhemoglobin consumes endothelium-derived NO and disrupts vasomotor balance. However, the administration of NO maintains vasomotor tone by converting oxyhemoglobin to methemoglobin, which does not consume endothelium-derived NO.

Effects of hemolysis and inhaled NO on hemodynamic responses to sodium nitroprusside, an infused NO donor. Sodium nitroprusside was administered to all animals during intervention experiments of the full-factorial study of inhaled NO and intravascular hemolysis in order to provide further evidence that plasma $\mathrm{Hb}$ released during hemolysis reacts with intravascular NO, thereby leading to systemic vasoconstriction. Sodium nitroprusside decreased SVRI and increased CI in a dose-dependent manner. The presence of hemolysis attenuated the expected vasodilatory response to escalating doses of sodium nitroprusside; however, concomitant inhalation of NO gas restored the expected nitroprusside response toward that of the nonhemolytic controls ( $P=0.005$ and 0.02 for SVRI and CI, respectively; Figure 7). Similar but not statistically significant patterns of response to increas- 

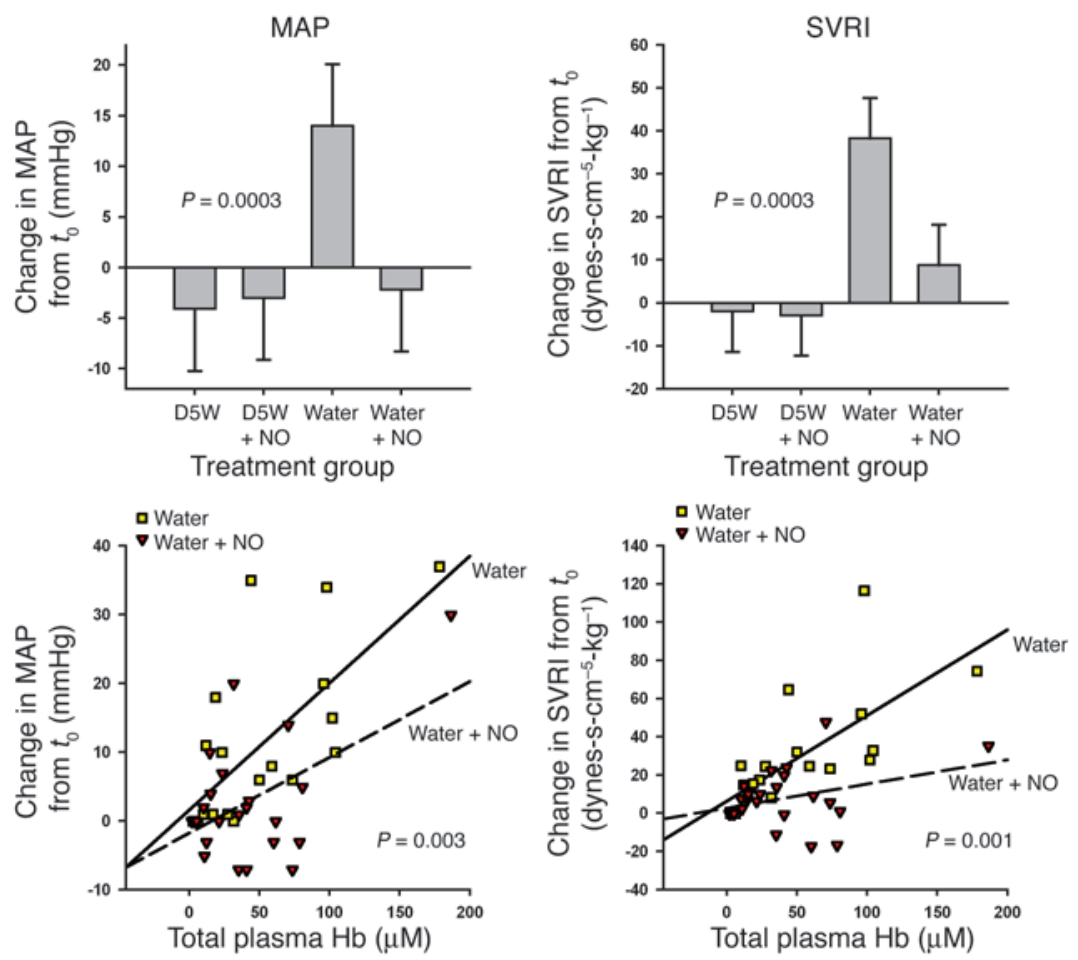

\section{Figure 4}

Relationship between total cell-free plasma $\mathrm{Hb}$ and the physiologic effects of hemolysis and inhaled NO. Upper panels: The difference in response from 0 to 6 hours between baseline and intervention studies for each of the 4 treatment groups is shown for MAP and SVRI. In animals receiving D5W (nonhemolyzing control groups), inhaled $\mathrm{NO}$ had no net effect on MAP and SVRI. Compared with these nonhemolyzing controls, free water-induced intravascular hemolysis caused significant increases in MAP and SVRI, which were attenuated by the concurrent inhalation of NO gas $(P=0.0003$ for interaction of $\mathrm{NO}$ and hemolysis for both variables). Lower panels: Relationship between change in MAP and SVRI and total plasma $\mathrm{Hb}$ levels (concentration in terms of heme groups) during the intervention studies in the hemolyzing groups (free water and free water plus NO groups). Despite similar total plasma $\mathrm{Hb}$ levels in these 2 groups, the relationships between change in MAP and SVRI and total plasma $\mathrm{Hb}$ levels were significantly different $(P=0.003$ and $P=0.001$, respectively). As total plasma $\mathrm{Hb}$ levels increased, MAP and SVRI increased more in the free water group than in the free water plus NO group. ing doses of sodium nitroprusside in the 4 treatment groups were also demonstrated for MAP, pulmonary artery pressure (PAP), heart rate (HR), central venous pressure (CVP), and pulmonary capillary wedge pressure (PCWP). In fact, all 7 hemodynamic variables demonstrated the expected ordered responses to nitroprusside $(P=0.008$ for $7 / 7$ variables having the same response pattern). These diminished responses of sodium nitroprusside during hemolysis support the hypothesis that cell-free plasma $\mathrm{Hb}$ consumes NO within the vasculature during intravascular hemolysis. These results may also, in part, be explained by the direct reaction of sodium nitroprusside with oxyhemoglobin (26).

Physiologic effects of Hb infusions are attenuated by inhaled NO. In a second model using direct infusions of canine $\mathrm{Hb}$ preparations, plasma $\mathrm{Hb}$ significantly increased SVRI and pulmonary vascular resistance index (PVRI), and inhaled NO attenuated $\mathrm{Hb}$-induced increases in SVRI and PVRI (Figure 8). In animals breathing air, plasma $\mathrm{Hb}$ remained predominantly oxyhemoglobin over the course of the infusion. In contrast, in animals breathing $\mathrm{NO}$, the plasma $\mathrm{Hb}$ was converted to predominantly methemoglobin over the course of the infusion. In the animals breathing NO, there was a higher concentration of methemoglobin in the systemic arterial circulation than in the pulmonary circulation (mean difference, $4.5 \%$; 95\% confidence interval, $-0.6 \%$ to $9.7 \% ; P=0.07)$, suggesting that the conversion of oxyhemoglobin to methemoglobin was occurring in the pulmonary circulation at the site of NO inhalation.

Figure 5
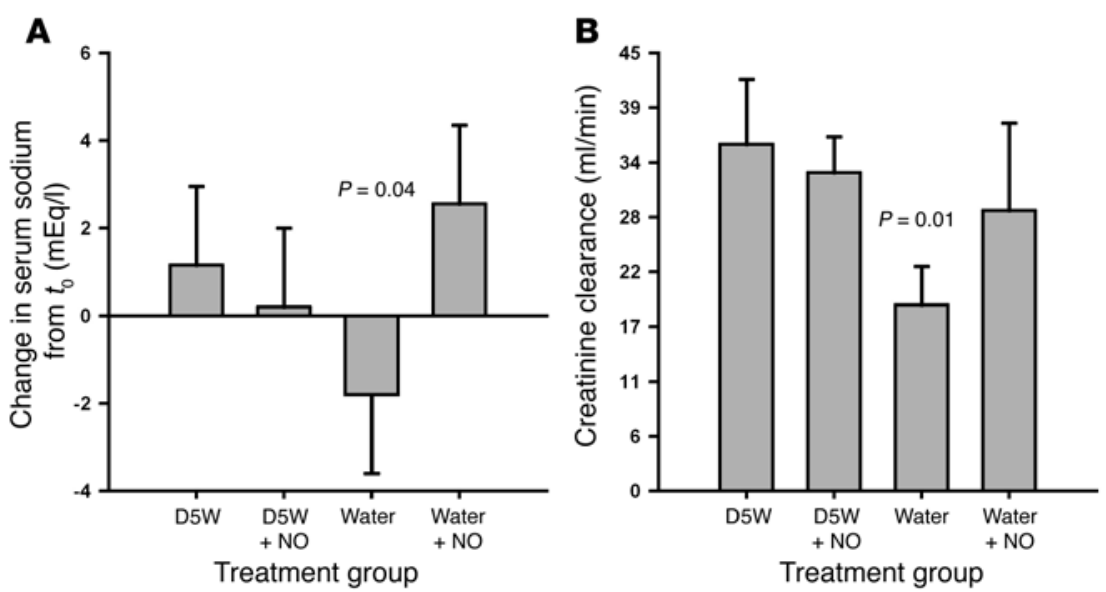

Effects of hemolysis and inhaled NO on renal function. (A) The difference in response from 0 to 6 hours between baseline and intervention studies for each of the 4 treatment groups is shown for serum sodium levels. Compared with infusion of D5W with or without NO, free water-induced intravascular hemolysis caused a significant impairment in the ability of the kidneys to compensate for hyponatremia, which was attenuated by the concurrent inhalation of NO $(P=0.04)$. (B) Six-hour creatinine clearance values during the intervention studies for each of the 4 treatment groups are shown. Based on a priori hypotheses, the creatinine clearance values ordered as expected, with the free water group having the lowest clearance, the D5W and D5W plus NO groups having the highest clearances, and the free water plus NO group having an intermediate clearance approaching that of the D5W and D5W plus NO groups $(P=0.01)$. 
A

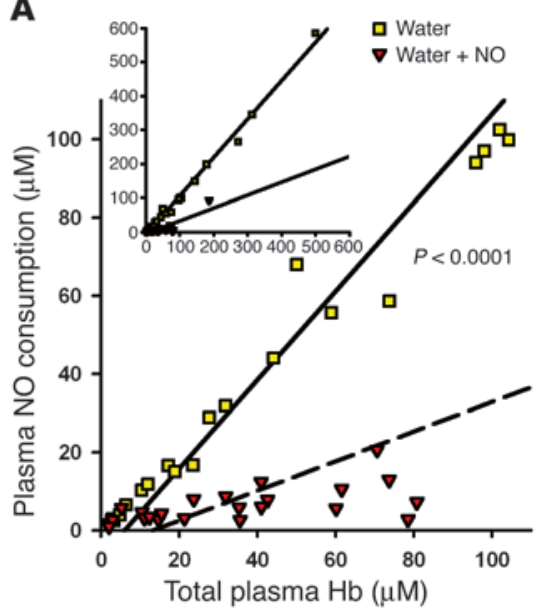

B
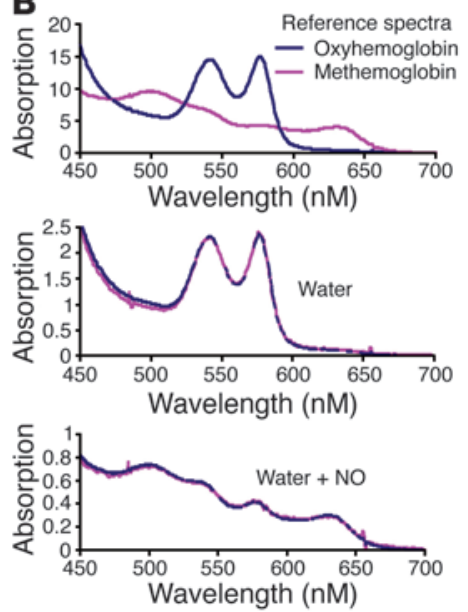

C

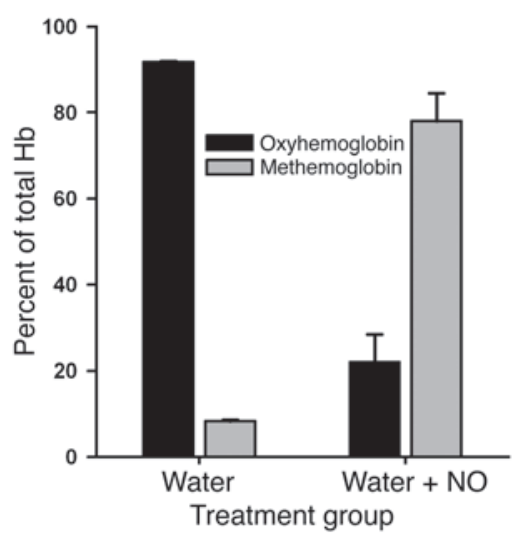

\section{Figure 6}

Plasma NO consumption and plasma Hb levels. (A) A significantly different relationship exists between plasma NO consumption and total plasma $\mathrm{Hb}$ levels (concentration in terms of heme groups) in the free water and free water plus NO groups $(P<0.0001)$. The inset demonstrates the relationships over the entire range of measured $\mathrm{Hb}$ levels, whereas the main graph focuses on the physiologic range of hemolysis in human disease states. (B) Spectral deconvolution of the plasma $\mathrm{Hb}$ species. The upper spectrum represents reference tracings for canine oxyhemoglobin and methemoglobin. The middle and lower spectra represent characteristic samples from the free water and free water plus NO treatment groups, respectively. (C) Total plasma $\mathrm{Hb}$ composition in the free water and free water plus NO groups was significantly different at 6 hours $(P=0.03)$. In the free water group, the plasma contained predominantly oxyhemoglobin. In contrast, in the free water plus NO group, the plasma contained predominantly methemoglobin.

in the first model, the total intravascular $\mathrm{Hb}$ level is unchanged; only the compartmentalization of $\mathrm{Hb}$ within the erythrocyte membrane is disrupted. These experiments demonstrated that hemolysis produces dose-dependent vasoconstriction and impaired renal function secondary to the stoichiometric oxidation of endogenous and exogenous NO by cell-free plasma oxyhemoglobin. Importantly, $80 \mathrm{ppm}$ inhaled NO gas oxidized $85-90 \%$ of the plasma oxyhemoglobin to methemoglobin, which inhibited endogenous NO scavenging, prevented systemic vasoconstriction, and restored responsiveness to systemically infused NO donors. This observation confirms that the observed physiological effects of hemolysis are directly mediated by the dioxygenation reaction of ferrous oxyhemoglobin and NO (Reaction 1). These studies provide evidence for the existence of a syndrome of hemolysis-associated endothelial dysfunction and suggest a potential therapeutic role of inhaled NO for iatrogenic, acquired, and hereditary hemolytic diseases. Specifically, the consumption and loss of NO activity during intravascular hemolysis may contribute to clinical signs and symptoms attributable to vasomotor instability that may be ameliorated by $\mathrm{NO}$ gas inhalation.

The levels of plasma Hb produced in the present study (with concentration expressed in terms of heme group) are within the ranges observed in human disease states. For example, in patients with sickle cell disease, the levels of plasma oxyheme range from 2 to $20 \mu \mathrm{M}$, with mean levels of $4 \mu \mathrm{M}$ (18). During vasoocclusive crisis, levels can rise to $20-40 \mu \mathrm{M}$ (27). Intravascular hemolysis in patients with paroxysmal nocturnal hemoglobinuria produces abdominal pain, gastric and esophageal dystonia, and erectile dysfunction, with levels of plasma $\mathrm{Hb}$ ranging from 30 to $120 \mu \mathrm{M}$ (in heme concentration) and up to $600 \mu \mathrm{M}$ during paroxysms of hemolysis (28). During cardiopulmonary bypass, the levels of plasma oxyheme can rise to $150 \mu \mathrm{M}(29-32)$. The present studies suggest that these pathologically relevant levels of hemoglobine- mia may be associated with endogenous NO inhibition, a vasopressor effect, and impaired organ function.

The biochemical and physiologic disturbances that occur during intravascular hemolysis are related to the disruption of diffusional barriers that typically regulate the reaction of intra-erythrocytic $\mathrm{Hb}$ with $\mathrm{NO}$ (11-17). Cell-free plasma $\mathrm{Hb}$ can react with endothelial cell-produced $\mathrm{NO}$ at nearly diffusion-limited rates, resulting in the rapid dioxygenation of $\mathrm{NO}$ by $\mathrm{Hb}$ with the formation of nitrate and methemoglobin (6-9). These rapid reactions greatly limit the diffusion of NO from endothelium to smooth muscle. Consequently, smooth muscle guanylyl cyclase is not activated, and vascular relaxation and vasodilation are inhibited (33). Additionally, cell-free plasma $\mathrm{Hb}$ can rapidly extravasate into the spaces between endothelial and smooth muscles cells and further scavenge NO, thereby prohibiting it from diffusing into smooth muscle cells (10). This mechanism is supported by the observed decrease in hypertensive effects of the Hb-based blood substitutes as molecular weight increases $(7,23)$ and the decrease in NO scavenging effects of the high-molecular-weight haptoglobin-Hb complex, compared with non-haptoglobin-bound $\mathrm{Hb}$ (34). We have proposed that the formation of Hb-haptoglobin high-molecularweight multimers serves to limit such extravasation during physiologic intravascular hemolysis (35).

$\mathrm{NO}$ scavenging by cell-free plasma $\mathrm{Hb}$ may have contributed to the increased morbidity and mortality observed in studies of stroma-free $\mathrm{Hb}$ artificial blood substitute solutions (7). The administration of the blood substitute solutions in preclinical and clinical trials led to pulmonary and systemic hypertension, increased systemic vascular resistance, decreased organ perfusion, gastrointestinal spasm and dysmotility, and death $(7,36-43)$. While studies of new generation Hb-based blood substitutes with heme pocket mutations designed to decrease the heme reactivity with NO have shown reduced vasopressor 

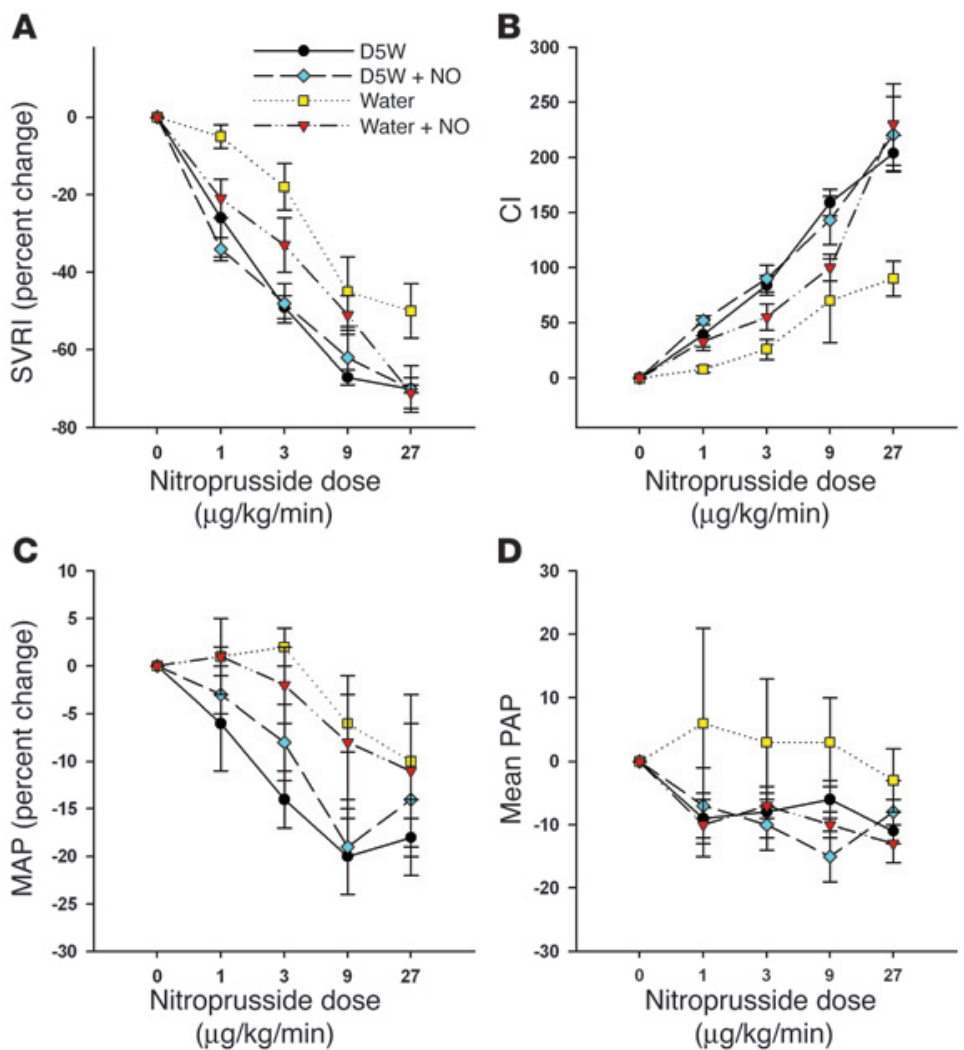

Figure 7

Physiologic effects of sodium nitroprusside during hemolysis with and without inhaled NO. Percent change in SVRI (A) and $\mathrm{Cl}(\mathbf{B})$ in response to increasing doses of sodium nitroprusside during the intervention studies for each of the 4 treatment groups. Compared with D5W and D5W plus NO, free water-induced hemolysis led to blunted hemodynamic effects of escalating doses of sodium nitroprusside, which were restored with inhaled NO therapy and oxidation of plasma $\mathrm{Hb}(P=0.005$ and $P=0.02$ for SVRI and $\mathrm{Cl}$, respectively). Similar but not statistically significant patterns of response to increasing doses of sodium nitroprusside in the 4 treatment groups were also demonstrated for MAP (C), PAP (D), heart rate, CVP, and PCWP. In fact, all 7 hemodynamic variables demonstrated the expected ordered responses to nitroprusside $(P=0.008$ for $7 / 7$ variables having the same response pattern).

effects (6), other investigators have suggested that the vasoconstrictor effects of $\mathrm{Hb}$-based blood substitutes occur secondarily to premature oxygen unloading at the precapillary sphincter $(24,25)$. The current study provides additional evidence that NO consumption by oxyhemoglobin is a mechanism of cellfree $\mathrm{Hb}$-mediated vasoconstriction and may contribute to the adverse effects observed in the blood substitute trials and during intravascular hemolysis in human disease.

In chronic hereditary hemolytic diseases such as sickle cell anemia, clinical complications such as pulmonary hypertension, priapism, leg ulcerations, and stroke may be in part related to repeated episodes of intravascular hemolysis with consequent increased NO scavenging, vasoconstriction, and end-organ hypoperfusion $(18,19,44)$. In patients with sickle cell disease, chronic hemolysis leads to the destruction of approximately $10 \%$ of the circulating erythrocytes every 24 hours, with $30 \%$ of this hemolysis estimated to be intravascular $(18,45)$. The Hb scavenging system in these patients becomes saturated, as indicated by undetectable plasma haptoglobin levels, and cell-free plasma $\mathrm{Hb}$ accumulates, as has been demonstrated by increased levels of plasma $\mathrm{Hb}$ in patients with sickle cell disease compared with normal patients (18). The plasma of these patients consumes significantly more NO than the plasma of normal patients, and the amount of NO consumption correlates with the plasma heme levels (18). Consistent with an NO-scavenging effect of increased cell-free plasma $\mathrm{Hb}$, the vasodilatory responses to nitroprusside, nitroglycerin, and other NO donors are significantly blunted in sickle cell patients and in sickle cell transgenic mouse models (18, 19, 46-48). Scavenging of NO by cell-free plasma Hb may be involved in the pathophysiologic vasculopathy and prothrombotic state that occur in many chronic hereditary hemolytic diseases such as sickle cell disease and thalassemia $(18,19,46-54)$ and acute hemolytic disease states, such as prolonged cardiopulmonary bypass, thrombotic thrombocytopenic purpura, and malaria. These studies provide further evidence that inhaled $\mathrm{NO}$ therapy may attenuate the NO-scavenging effects of cell-free plasma $\mathrm{Hb}$ and may be able to block the pathophysiologic changes that occur during iatrogenic (cardiopulmonary bypass) or disease-specific (sickle cell pain crisis, malaria, etc.) intravascular hemolysis in many human diseases. Further research is required to assess the contribution of hemolysis and therapeutic utility of inhaled NO therapy in hereditary and iatrogenic hemolytic disorders.

In conclusion, these data provide controlled in vivo evidence that NO scavenging by cell-free plasma $\mathrm{Hb}$ during intravascular hemolysis disrupts endothelial NOdependent vasomotor function and produces systemic physiologic changes and organ dysfunction, which are attenuated by inhaled NO therapy. These biochemical and physiological studies support the existence of a syndrome of hemolysis-associated endothelial dysfunction, which may contribute to the vasculopathy of hereditary, acquired, and iatrogenic hemolytic states. Furthermore, these studies support a potential therapeutic role for $\mathrm{NO}$ donor agents in preventing the end-organ injury associated with these disease states.

\section{Methods}

Experimental design. All experiments were approved by the Animal Care and Use Committee of the Clinical Center of the NIH. In all, 32 purpose-bred beagles (12-28 months, 9-12 kg) were studied.

All procedures were performed after the induction of anesthesia with halothane (1-4\%) and the initiation of mechanical ventilation. Once all procedures were finished, the halogenated gas was terminated, and $100 \%$ oxygen administered until the dog emerged from anesthesia. The dog was then maintained on continuous sedation with intravenous medetomidine $(2-5 \mu \mathrm{g} / \mathrm{kg} / \mathrm{h})$ and received continuous intravenous analgesia with fentanyl $(5-15 \mu \mathrm{g} / \mathrm{kg} / \mathrm{h})$. The animals were monitored continuously, and all signs of pain and distress were evaluated immediately and the infusions adjusted appropriately.

Model development. A canine model of free water-induced intravascular hemolysis was developed to determine the optimal dose of free water administration that produced clinically relevant levels of cell-free plasma $\mathrm{Hb}$ (20-300 $\mu \mathrm{M}$ heme), simulating an acute hemolytic episode. Increasing rates of free water $(2,4,8,16 \mathrm{ml} / \mathrm{kg} / \mathrm{h})$ were administered intravenously through 
A
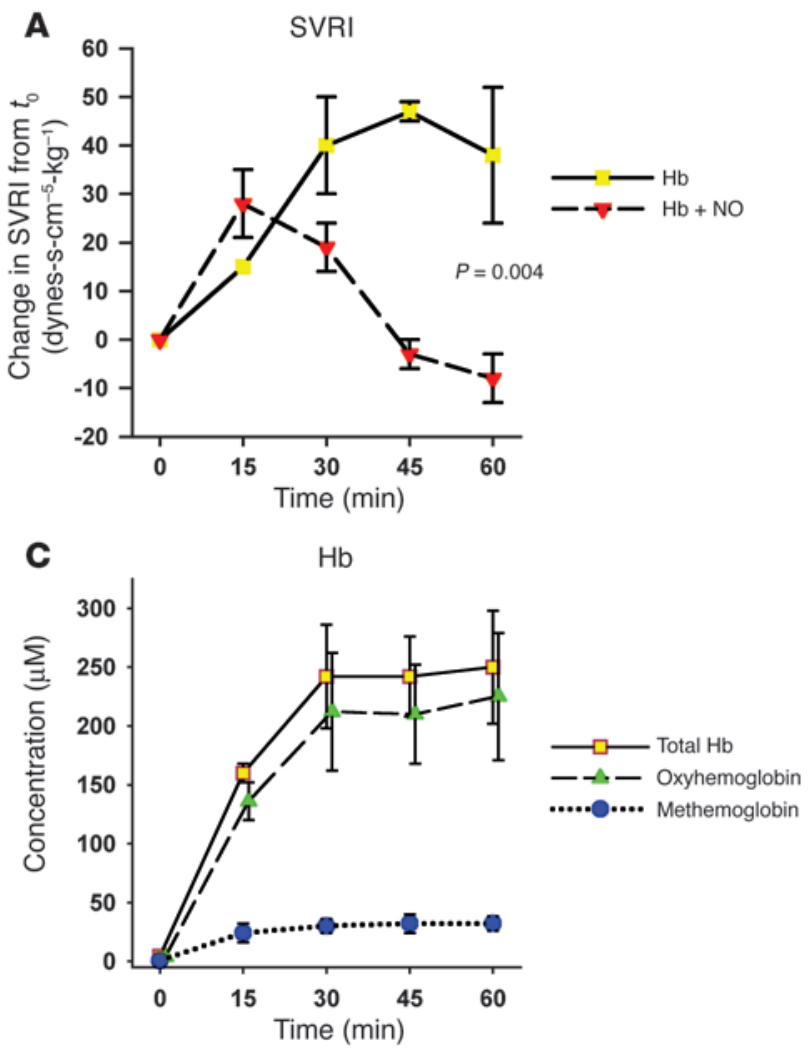
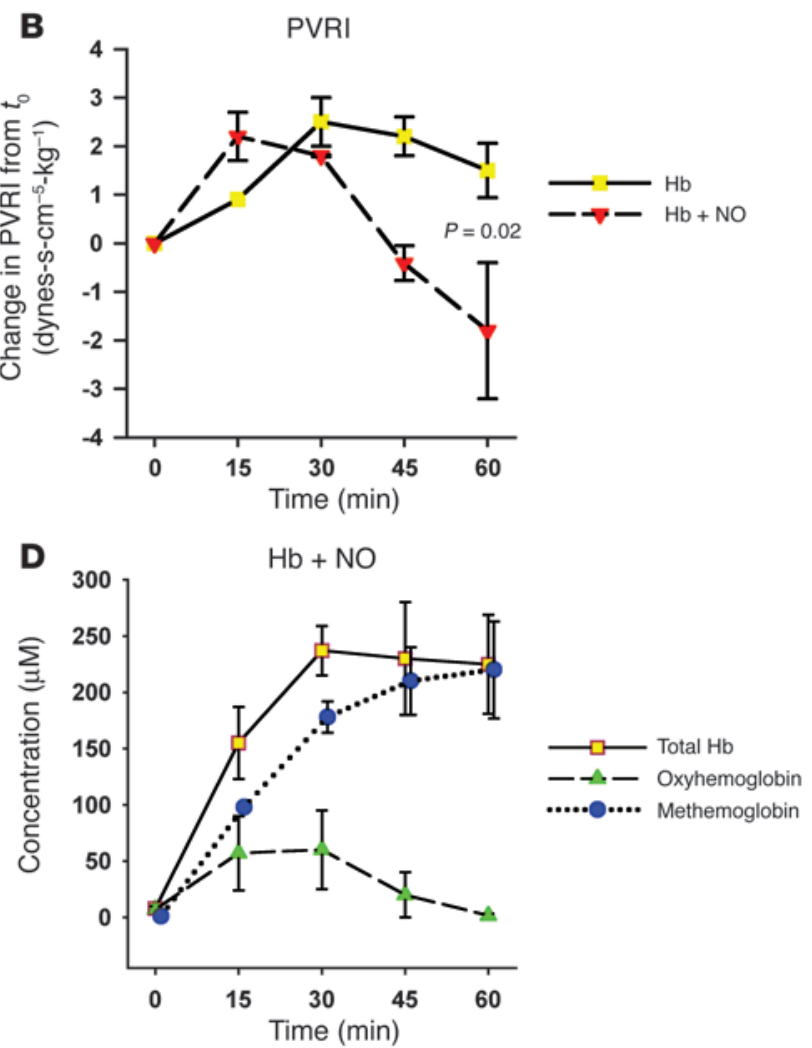

Figure 8

Effects of $\mathrm{Hb}$ infusions with and without inhaled NO. Infusion of cell-free $\mathrm{Hb}$ led to increases in SVRI and pulmonary vascular resistance index that were attenuated by inhaled NO (A and B). In animals breathing air $(n=2)$, the cell-free Hb remained predominantly oxyhemoglobin $(\mathbf{C})$. In contrast, in animals breathing $\mathrm{NO}(n=2)$, the cell-free $\mathrm{Hb}$ was converted to methemoglobin (D).

a central venous catheter to 4 animals over 6 hours in order to characterize the relationship between the rate of free water infusion and plasma concentration of cell-free $\mathrm{Hb}$. Subsequent experiments were performed $(n=8)$ to determine the ideal control fluid to be used in the full-factorial design as well as to delineate the biochemical and pathophysiologic effects of acute intravascular hemolysis in our model. In these pilot experiments, each animal received a 6 -hour baseline infusion of 1 of 2 potential control group fluids through the central venous catheter $(n=4,0.9 \%$ sodium chloride; $n=4$, D5W) followed 1 week later by a 6 -hour infusion of free water through a central venous catheter. Based on these pilot experiments, a free water infusion rate of $16 \mathrm{ml} / \mathrm{kg} / \mathrm{h}$ was chosen to achieve the desired rate of intravascular hemolysis. While there was no difference in the hemodynamic responses to either control fluid, $0.9 \%$ sodium chloride or D5W, we chose the latter to control for any potential hypotonic effects of free water infusion.

Full-factorial study design. This set of experiments examined the effects of inhaled NO gas and oxidation of plasma oxyhemoglobin to methemoglobin on the biochemical and physiologic changes caused by intravascular hemolysis. Paired experiments were performed in 20 tracheostomized animals (5 per group) and included a 6-hour baseline study with infusion of D5W through a central venous catheter followed 1 week later by a 6-hour intervention study with infusion of either D5W or free water through a central venous catheter with or without inhaled NO (Figure 1). All animals received $21 \%$ fraction inspired oxygen $\left(\mathrm{FiO}_{2}\right)$ through tracheostomy using an air/oxygen blender. Eighty ppm NO gas with $21 \% \mathrm{FiO}_{2}$ was simultaneously administered to animals randomized to receive NO therapy. In order to determine the vascular responsiveness to exogenous
$\mathrm{NO}$ in the presence and absence of hemolysis and inhaled NO, all animals received a 20 -minute infusion of escalating doses of sodium nitroprusside, a direct NO donor $(1,3,9$, and $27 \mu \mathrm{g} / \mathrm{kg} / \mathrm{min})$ at 5 -minute intervals prior to the conclusion of the study.

Data collection. For all studies, femoral arterial (20-gauge) and external jugular venous (8 French) catheters (Maxxim Medical) were placed percutaneously under anesthesia using aseptic sterile technique. MAP and HR were obtained from the femoral artery catheter tracing. Additionally, a pulmonary artery thermodilution catheter (7 French; Abbott Critical Care Systems, Abbott Laboratories) was introduced through the external jugular vein catheter in order to obtain cardiac output (CO), CI, PAP, PCWP, and CVP measurements. Urinary catheters (12 French) were placed during each study under anesthesia using aseptic technique. All catheter placements were performed under general anesthesia. At the end of the first week's fluid control experiments, all catheters were removed, and the animals recovered. At the end of the second week's intervention experiments, all animals were euthanized.

In all experiments, hemodynamic measurements (MAP, CVP, PAP, CO, and PCWP) and laboratory studies (analysis of Hct, Hb, and serum chemistries; arterial blood gas analysis; spectrophotometric-based quantification of cell-free $\mathrm{Hb}$ concentration; and chemiluminescence-based assays of NO consumption) were obtained at 0-, 1.5-, 3.0-, 4.5-, and 6.0-hour time points. Urine was collected from 0 to 6 hours to measure 6 -hour creatinine clearances. Hemodynamic measurements were also obtained at the end of each 5-minute interval of sodium nitroprusside infusion at doses of $1,3,9$, and $27 \mu \mathrm{g} / \mathrm{kg} / \mathrm{min}$. 
Confirmatory $\mathrm{Hb}$ infusion experiments. The contribution of cell-free $\mathrm{Hb}$ to the physiologic findings in our water infusion model cannot be completely isolated, because this model simulates an acute episode of intravascular hemolysis with the release of erythrocyte membrane and all cellular contents into the plasma. Also, the hypotonic swelling of the erythrocytes in our model does not always lead to cell lysis and may have contributed to the physiologic alterations in our model. Therefore, to confirm and extend the findings of the intravascular water infusion model, additional experiments were performed with infusion of $99 \%$ pure $\mathrm{Hb}$ solutions directly into the animals. Canine whole blood was collected and diluted with PBS buffer (1:2). The blood was frozen and thawed 3 times and then centrifuged at $82,000 \mathrm{~g}$ for 1 hour at $4{ }^{\circ} \mathrm{C}$ to remove the membrane fraction. The supernatant was collected and filtered $(0.22 \mu \mathrm{M})$. Hb concentrations were measured, and the solutions were stored at $4^{\circ} \mathrm{C}$ until infusion. Prior to infusion, each solution underwent a second filtration $(0.22 \mu \mathrm{M})$. These additional experiments allowed for direct measurements of the physiologic and biochemical effects of cell-free $\mathrm{Hb}$ infusion with and without inhaled $\mathrm{NO}$ exposure. $\mathrm{Hb}$ solutions were infused into animals breathing air $(n=2)$ or $80 \mathrm{ppm} \mathrm{NO}(n=2)$ for 1 hour. Serial physiologic and biochemical measurements were obtained.

Plasma Hb, NO consumption, and haptoglobin assays. The ability of plasma to consume $\mathrm{NO}$ was measured with a previously published and validated NO consumption assay using an NO chemiluminescence analyzer (Sievers 280i NO analyzer; GE Analytical Instruments) as previously described (35). Total plasma $\mathrm{Hb}$ concentration (expressed in terms of heme groups; division by 4 gives $\mathrm{Hb}$ concentration) was measured by visible absorbance spectrophotometry (HP8453 UV-Vis Diode Array Spectrophotometer; Hewlett-Packard). The concentrations of oxyhemoglobin and methemoglobin were analyzed by deconvoluting the spectrum into components from basis spectra of canine $\mathrm{Hb}$ composed of oxyhemoglobin and methemoglobin in PBS buffer using a least-square method similar to that previously described, with subtraction of background plasma scattering (55). Plasma haptoglobin levels were measured using a polyclonal anti-human haptoglobin antibody (Sigma-Aldrich) as previously described (18).

Statistics. Pilot data were analyzed using ANOVA, with main effects for study (baseline and hemolysis), time, and animal, and a 2 -way interaction between study and time. The full-factorial treatment experiments were analyzed using ANOVA, with main effects for study (baseline and intervention), 4 treatment arms, time, and animal (nested within each of the 4 arms). Two- and 3-way interactions, not involving animal, were included in the model. Analysis of responses to sodium nitroprusside were performed using ANOVA on percent change in hemodynamic variables with increasing dose in the intervention study with main effects for the 4 treatment arms, dose, and animal (nested within each of the 4 arms). Two-way interactions, not involving animal, were included in the model. Analysis of differences in creatinine clearance during the intervention study was performed using a nonparametric test for ordered alternatives based on the a priori hypothesis that free water would decrease creatinine clearance and that inhaled NO would attenuate this decrease (Jonckheere-Terpstra test) (56). Comparisons of the 2 groups of animals in the confirmatory $\mathrm{Hb}$ infusion experiments were performed with ANOVA with main effects for treatment group and time and a 2-way interaction term. All values are represented in the figures as mean $\pm \mathrm{SEM}$, and all $\mathrm{Hb}$ concentrations are expressed in terms of heme groups. $P<0.05$ was considered statistically significant.

\section{Acknowledgments}

This study was funded by intramural sources at the NHLBI and the Critical Care Medicine Department, Clinical Center at the $\mathrm{NIH}$. We gratefully thank INO Therapeutics for supplying the NO gas and delivery systems for this study.

Received for publication March 14, 2005, and accepted in revised form September 13, 2005.

Address correspondence to: Mark T. Gladwin, Vascular Medicine Branch, NHLBI, Critical Medicine Department, Clinical Center, National Institutes of Health, Clinical Research Center, Building 10-CRC, Room 5-5140, Bethesda, Maryland 20892, USA. Phone: (301) 435-2324; Fax: (301) 451-7091; E-mail: mgladwin@nih.gov.

Peter C. Minneci and Katherine J. Deans contributed equally to this work.

Mark T. Gladwin and Steven B. Solomon are co-senior authors.
1. Furchgott, R.F., and Zawadzki, J.V. 1980. The obligatory role of endothelial cells in the relaxation of arterial smooth muscle by acetylcholine. Nature. 288:373-376.

2. Ignarro, L.J., Buga, G.M., Wood, K.S., Byrns, R.E., and Chaudhuri, G. 1987. Endothelium-derived relaxing factor produced and released from artery and vein is nitric oxide. Proc. Natl. Acad. Sci. U. S. A. 84:9265-9269.

3. Ignarro, L.J., Byrns, R.E., Buga, G.M., and Wood, K.S. 1987. Endothelium-derived relaxing factor from pulmonary artery and vein possesses pharmacologic and chemical properties identical to those of nitric oxide radical. Circ. Res. 61:866-879.

4. Palmer, R.M., Ferrige, A.G., and Moncada, S. 1987. Nitric oxide release accounts for the biological activity of endothelium-derived relaxing factor. Nature. 327:524-526.

5. Palmer, R.M., Ashton, D.S., and Moncada, S. 1988. Vascular endothelial cells synthesize nitric oxide from L-arginine. Nature. 333:664-666.

6. Doherty, D.H., et al. 1998. Rate of reaction with nitric oxide determines the hypertensive effect of cell-free hemoglobin. Nat. Biotechnol. 16:672-676.

7. Dou, Y., Maillett, D.H., Eich, R.F., and Olson, J.S. 2002. Myoglobin as a model system for designing heme protein based blood substitutes. Biophys. Chem. 98:127-148.

8. Gladwin, M.T., Lancaster, J.R., Freeman, B.A., and
Schechter, A.N. 2003. Nitric oxide's reactions with hemoglobin: a view through the SNO-storm. Nat. Med. 9:496-500.

9. Schechter, A.N., and Gladwin, M.T. 2003. Hemoglobin and the paracrine and endocrine functions of nitric oxide. N. Engl. J. Med. 348:1483-1485.

10. Nakai, K., et al. 1996. Inhibition of endotheliumdependent relaxation by hemoglobin in rabbit aortic strips: comparison between acellular hemoglobin derivatives and cellular hemoglobins. J. Cardiovasc. Pharmacol. 28:115-123.

11. Vaughn, M.W., Kuo, L., and Liao, J.C. 1998. Effective diffusion distance of nitric oxide in the microcirculation. Am. J. Physiol. 274:H1705-H1714.

12. Butler, A.R., Megson, I.L., and Wright, P.G. 1998. Diffusion of nitric oxide and scavenging by blood in the vasculature. Biochim. Biophys. Acta. 1425: $168-176$.

13. Liao, J.C., Hein, T.W., Vaughn, M.W., Huang, K.T., and Kuo, L. 1999. Intravascular flow decreases erythrocyte consumption of nitric oxide. Proc. Natl. Acad. Sci. U. S. A. 96:8757-8761.

14. Coin, J.T., and Olson, J.S. 1979. The rate of oxygen uptake by human red blood cells. J. Biol. Chem. 254:1178-1190.

15. Liu, X., et al. 1998. Diffusion-limited reaction of free nitric oxide with erythrocytes. J. Biol. Chem. 273:18709-18713.

16. Vaughn, M.W., Huang, K.T., Kuo, L., and Liao,
J.C. 2000. Erythrocytes possess an intrinsic barrier to nitric oxide consumption. J. Biol. Chem. 275:2342-2348.

17. Huang, K.T., et al. 2001. Modulation of nitric oxide bioavailability by erythrocytes. Proc. Natl. Acad. Sci. U. S. A. 98:11771-11776.

18. Reiter, C.D., et al. 2002. Cell-free hemoglobin limits nitric oxide bioavailability in sickle-cell disease. Nat. Med. 8:1383-1389.

19. Kaul, D.K., Liu, X.D., Chang, H.Y., Nagel, R.L., and Fabry, M.E. 2004. Effect of fetal hemoglobin on microvascular regulation in sickle transgenicknockout mice. J. Clin. Invest. 114:1136-1145. doi:10.1172/JCI200421633.

20. Gow, A.J., Luchsinger, B.P., Pawloski, J.R., Singel, D.J., and Stamler, J.S. 1999. The oxyhemoglobin reaction of nitric oxide. Proc. Natl. Acad. Sci. U. S. A. 96:9027-9032.

21. Gaston, B.M., and Hare, J.M. 2003. Hemoglobin and nitric oxide [letter]. N. Engl. J. Med. 349:402-405; author reply 402-405.

22. Pawloski, J.R. 2003. Hemoglobin and nitric oxide [letter]. N. Engl. J. Med. 349:402-405; author reply 402-405.

23. Olson, J.S., et al. 2004. NO scavenging and the hypertensive effect of hemoglobin-based blood substitutes. Free Radic. Biol. Med. 36:685-697.

24. Rohlfs, R.J., et al. 1998. Arterial blood pressure responses to cell-free hemoglobin solutions 
and the reaction with nitric oxide. J. Biol. Chem. 273:12128-12134.

25. McCarthy, M.R., Vandegriff, K.D., and Winslow, R.M. 2001. The role of facilitated diffusion in oxygen transport by cell-free hemoglobins: implications for the design of hemoglobin-based oxygen carriers. Biophys. Chem. 92:103-117.

26. Kruszyna, H., Kruszyna, R., Rochelle, L.G., Smith, R.P., and Wilcox, D.E. 1993. Effects of temperature, oxygen, heme ligands and sulfhydryl alkylation on the reactions of nitroprusside and nitroglycerin with hemoglobin. Biochem. Pharmacol. 46:95-102.

27. Naumann, H.N., Diggs, L.W., Barreras, L., and Williams, B.J. 1971. Plasma hemoglobin and hemoglobin fractions in sickle cell crisis. Am. J. Clin. Pathol. 56:137-147.

28. Hartmann, R.C., Jenkins, D.E., Jr., McKee, L.C., and Heyssel, R.M. 1966. Paroxysmal nocturnal hemoglobinuria: clinical and laboratory studies relating to iron metabolism and therapy with androgen and iron. Medicine (Baltimore). 45:331-363.

29. Davis, C.L., Kausz, A.T., Zager, R.A., Kharasch, E.D., and Cochran, R.P. 1999. Acute renal failure after cardiopulmonary bypass in related to decreased serum ferritin levels. J. Am. Soc. Nephrol. 10:2396-2402.

30. Murakami, F., et al. 1997. Clinical study of totally roller pumpless cardiopulmonary bypass system. Artif. Organs. 21:803-807.

31. Pepper, J.R., Mumby, S., and Gutteridge, J.M. 1994. Transient iron-overload with bleomycin-detectable iron present during cardiopulmonary bypass surgery. Free Radic. Res. 21:53-58.

32. Shimono, T., et al. 1997. Silicone-coated polypropylene hollow-fiber oxygenator: experimental evaluation and preliminary clinical use. Ann. Thorac. Surg. 63:1730-1736.

33. Pohl, U., and Lamontagne, D. 1991. Impaired tissue perfusion after inhibition of endothelium-derived nitric oxide. Basic Res. Cardiol. 86(Suppl. 2):97-105.

34. Edwards, D.H., Griffith, T.M., Ryley, H.C., and
Henderson, A.H. 1986. Haptoglobin-haemoglobin complex in human plasma inhibits endothelium dependent relaxation: evidence that endothelium derived relaxing factor acts as a local autocoid. Cardiovasc. Res. 20:549-556.

35. Wang, X., et al. 2004. Biological activity of nitric oxide in the plasmatic compartment. Proc. Natl. Acad. Sci. U. S. A. 101:11477-11482.

36. Winslow, R.M. 2000. alphaalpha-crosslinked hemoglobin: was failure predicted by preclinical testing? Vox Sang. 79:1-20.

37. Hess, J.R., MacDonald, V.W., and Brinkley, W.W. 1993. Systemic and pulmonary hypertension after resuscitation with cell-free hemoglobin. J. Appl. Physiol. 74:1769-1778.

38. Hess, J.R., Macdonald, V.W., Gomez, C.S., and Coppes, V. 1994. Increased vascular resistance with hemoglobin-based oxygen carriers. Artif. Cells Blood Substit. Immobil. Biotechnol. 22:361-372.

39. LaMuraglia, G.M., et al. 2000. The reduction of the allogenic transfusion requirement in aortic surgery with a hemoglobin-based solution. J. Vasc. Surg. 31:299-308.

40. Lamy, M.L., et al. 2000. Randomized trial of diaspirin cross-linked hemoglobin solution as an alternative to blood transfusion after cardiac surgery. The DCLHb Cardiac Surgery Trial Collaborative Group. Anesthesiology. 92:646-656.

41. Przybelski, R.J., et al. 1996. Phase I study of the safety and pharmacologic effects of diaspirin cross-linked hemoglobin solution. Crit. Care Med. 24:1993-2000.

42. Savitsky,J.P., Doczi,J., Black, J., and Arnold,J.D. 1978. A clinical safety trial of stroma-free hemoglobin. Clin. Pharmacol. Ther. 23:73-80.

43. Sloan, E.P., et al. 1999. Diaspirin cross-linked hemoglobin (DCLHb) in the treatment of severe traumatic hemorrhagic shock: a randomized controlled efficacy trial. JAMA. 282:1857-1864.

44. Nolan, V.G., Wyszynski, D.F., Farrer, L.A., and Stein- berg, M.H. 2005. Hemolysis associated priapism in sickle cell disease. Blood. doi:10.1182/blood-200504-1591.

45. Bensinger, T.A., and Gillette, P.N. 1974. Hemolysis in sickle cell disease. Arch. Intern. Med. 133:624-631.

46. Kaul, D.K., Liu, X.D., Fabry, M.E., and Nagel, R.L. 2000. Impaired nitric oxide-mediated vasodilation in transgenic sickle mouse. Am. J. Physiol. Heart Circ. Physiol. 278:H1799-H1806.

47. Eberhardt, R.T., et al. 2003. Sickle cell anemia is associated with reduced nitric oxide bioactivity in peripheral conduit and resistance vessels. Am. J. Hematol. 74:104-111.

48. Nath, K.A., et al. 2000. Mechanisms of vascular instability in a transgenic mouse model of sickle cell disease. Am. J. Physiol. Regul. Integr. Comp. Physiol. 279:R1949-R1955.

49. Aessopos, A., et al. 1995. Pulmonary hypertension and right heart failure in patients with beta- thalassemia intermedia. Chest. 107:50-53.

50. Du, Z.D., Roguin, N., Milgram, E., Saab, K., and Koren, A. 1997. Pulmonary hypertension in patients with thalassemia major. Am. Heart J. 134:532-537.

51. Derchi, G., et al. 1999. Pulmonary hypertension in patients with thalassemia major [letter]. Am. HeartJ. 138:384.

52. Grisaru, D., et al. 1990. Cardiopulmonary assessment in beta-thalassemia major. Chest. 98:1138-1142.

53. Koren, A., Garty, I., Antonelli, D., and Katzuni, E. 1987. Right ventricular cardiac dysfunction in betathalassemia major. Am. J. Dis. Child. 141:93-96.

54. Zakynthinos, E., et al. 2001. Pulmonary hypertension, interstitial lung fibrosis, and lung iron deposition in thalassaemia major. Thorax. 56:737-739.

55. Huang, Z., et al. 2001. Nitric oxide binding to oxygenated hemoglobin under physiological conditions. Biochim. Biophys. Acta. 1568:252-260.

56. Lehmann, E.L. 1975. Nonparametrics: statistical methods based on ranks. Holden-Day. San Francisco, California, USA. 457 pp. 